\title{
Experimental Spin Ratchet
}

Marius V. Costache and Sergio O. Valenzuela

Science 330, 1645 (2010)

DOI: $10.1126 /$ science. 1196228

\section{Recommended with a Commentary by Roland Kawakami, University of California at Riverside, USA}

One of the appealing aspects of spin transport and spin electronics is the prospect of utilizing pure spin currents in the absence of charge currents. For example, a pure spin current can be made by having spin-up electrons transporting to the right and an equal number of spin-down electrons transporting to the left. In this case, there is no net charge current, but there is an overall spin-up angular momentum current to the right (we take the convention to call this "spin current to the right" based on the flow of the spin-up species). Experimentally, pure spin currents have been generated by the spin Hall effect and in the non-local spin transport geometry. However, both of these must be driven by a DC current and the resulting pure spin current is proportional to this applied DC current.

An alternative approach proposed a few years ago is to develop a "spin ratchet" in which the flow of electrons depends on both the electrical bias and on the spin species. Figure 1 shows a

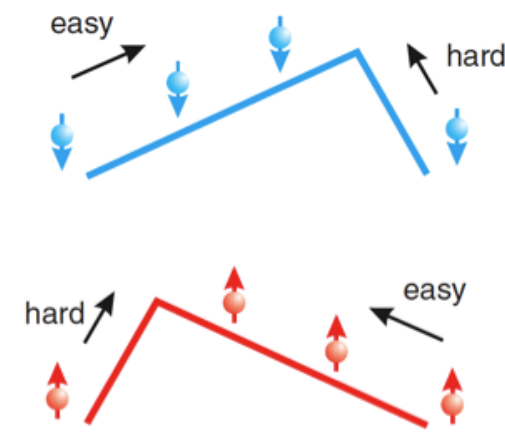

Fig. 1: Spin ratchet situation in which it is easier for spin-down electrons to flow to the right and for spin-up electrons to flow to the left. Thus, the application of an AC electrical bias can produce a DC spin current to the left. The first theoretical proposals for a spin ratchet were based on Rashba-like spin-orbit coupling in semiconductor nanowires [1]. Because the spin-orbit field depends linearly on $k$, this creates a spin-asymmetry which is needed for the spin ratchet as shown in Figure 1.

The work by Costache and Valenzuela is the first experimental demonstration of a spin ratchet. Interestingly, it does not operate through the spin-orbit interation, but rather by a different mechanism in a superconducting single electron transistor (SET). Here, I present a highly simplified picture of the mechanism of the spin ratchet. The essential ingredients are a superconducting SET, asymmetric tunneling contacts to metal electrodes, and an applied magnetic field. Because the island is superconducting, the odd states of the SET (call it $n=1$ ) have an unpaired electron, so that the energy is raised by the superconducting gap $\Delta$. The SET energetics are shown in Figure $2 \mathrm{a}$ with higher $\mathrm{E}$ for $n=1$ compared to $n=0$ and $n=2$. Applying a magnetic field (Figure 2b) produces a Zeeman splitting which can be adjusted to make a simultaneous degeneracy between the $n=0, n=2$, and the spin-down $n=1$ state ( $n=1^{\downarrow}$ ), which promotes conduction through the SET.

In this degenerate regime, one then considers the various tunneling rates. It is argued that the rate to go from an even state to an odd state $\left(\Gamma^{e \rightarrow o}\right)$ is much larger than
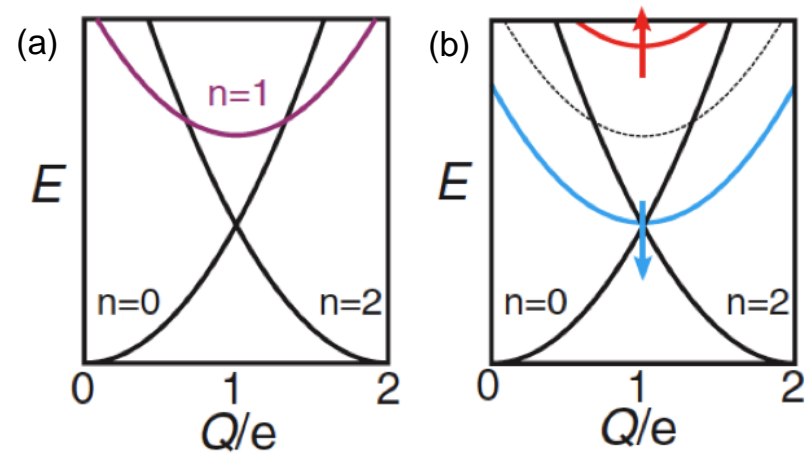

Fig. 2: SET energetics 
the rate to go from an odd state to and even state $\left(\Gamma^{o \rightarrow e}\right)$. Therefore, while the $n=0, n=1^{\downarrow}$, and $n=2$ are degenerate, the dynamics makes the system to be usually in the $n=1^{\downarrow}$ state. For the spin ratchet, it is required to have asymmetric tunneling so that $\Gamma^{o \rightarrow e}$ is different for the left and right contacts, say $\Gamma_{R}^{o \rightarrow e}>\Gamma_{L}^{o \rightarrow e}$. Figure 3a shows the tunneling sequence when bias is applied to flow electrons to the right, and Figure $3 \mathrm{~b}$ shows the tunneling sequence when bias is applied to flow electrons to the left. Because $\Gamma_{R}^{o \rightarrow e}>\Gamma_{L}^{o \rightarrow e}$, the tunneling sequence is primarily determined by the right contact. For Figure 3a, the spin down electron will flow off to the right electrode to take the system to the $n=0$ state; then a spin down electron will flow on from the left electrode to get back to the $n=1^{\downarrow}$ state. The electron current is to the right and the spin current is to the left. For Figure 3b, a spin up electron will enter from the right electrode to take the system to the $n=2$ state; then the spin up electron will flow off to the left electrode to get back to the $n=1^{\downarrow}$ state. The electron current is to the left and the spin current is to the left. Thus, either bias produces a spin current to the left and the ratchet action is achieved. Experimentally, this behavior is demonstrated using ferromagnetic electrodes and observing different conductance peak heights for positive and negative bias.

(a)
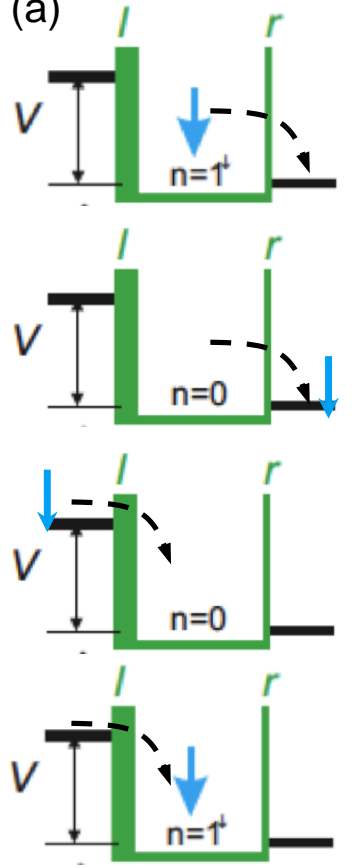

electron motion

spin current
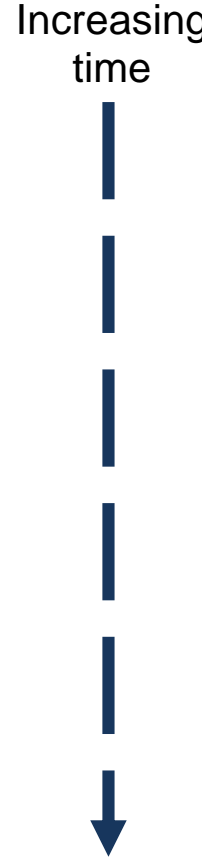

Fig. 3: Spin flow determined by the lower resistance contact

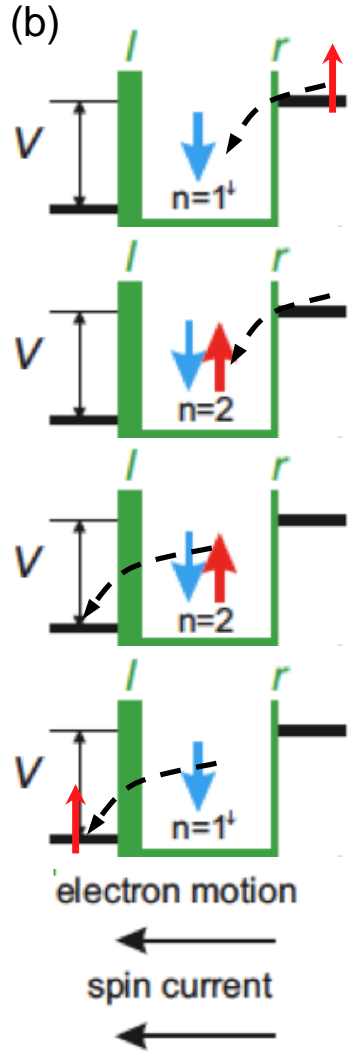

[1] Sergey Smirnov, Dario Bercioux, Milena Grifoni, and Klaus Richter, "Quantum Dissipative Rashba Spin Ratchets," Phys. Rev. Lett. 100, 230601 (2008). 